\title{
Cash transfers in context. An anthropological perspective
}

\section{Vincent Geronimi}

\section{OpenEdition}

\section{Journals}

Édition électronique

URL : https://journals.openedition.org/anthropodev/1051

DOI : 10.4000/anthropodev. 1051

ISSN : 2553-1719

\section{Éditeur}

Presses universitaires de Louvain

Édition imprimée

Date de publication : 1 décembre 2020

Pagination : 189-192

ISBN : 978-2-39061-078-6

ISSN : 2276-2019

\section{Référence électronique}

Vincent Geronimi, « Cash transfers in context. An anthropological perspective », Anthropologie \&

développement [En ligne], 51 | 2020, mis en ligne le 01 décembre 2020, consulté le 02 février 2022

URL : http://journals.openedition.org/anthropodev/1051; DOI : https://doi.org/10.4000/anthropodev. 1051

La revue Anthropologie \& développement est mise à disposition selon les termes de la Licence Creative Commons Attribution 4.0 International. 


\title{
Cash transfers in context. An anthropological perspective
}

\author{
Jean-Pierre Olivier de Sardan et Emmanuelle Piccoli, 2018, New York, \\ Oxford, Berghahn Books, 360 p.
}

Vincent Geronimi

Après un rapide résumé des principaux apports de l'ouvrage, cette note de lecture présente quelques pistes ouvertes à la réflexion pour les communautés des économistes (le rédacteur de cette note appartient à ces tribus) et des anthropologues du développement.

L'ouvrage dirigé par Jean-Pierre Olivier de Sardan et Emmanuelle Piccoli apporte une analyse approfondie des dispositifs de transferts monétaires (cash transfers ou CT) à travers une approche anthropologique (Olivier de sardan, 1995), mobilisant plusieurs études de cas, en Afrique et en Amérique du Sud. Il s'agit d'une contribution originale que les chercheurs, experts, praticiens, etc. impliqués dans les questions de développement économique et de changement social liront avec beaucoup d'intérêt. Les politiques et programmes de $\mathrm{CT}$ constituent un thème particulièrement important car ils représentent depuis plusieurs années un modèle à suivre pour les institutions et organisations de développement, avec une large diffusion internationale. En mobilisant la méthode anthropologique à partir d'études de terrain, les analyses rassemblées dans l'ouvrage permettent de disposer d'une vision alternative à celle, normative et simplificatrice, des "bonnes politiques» et "bonnes pratiques" portées par la plupart des institutions internationales, publiques comme privées. Ces bonnes politiques et bonnes pratiques à visée universelle sont souvent, comme dans le cas des CT, basées sur des études quantitatives (essai randomisé contrôlé (ERC), économétrie) participant à la définition d'un mécanisme, et sur un récit construit à partir d'expériences initiales, contribuant à définir un "modèle voyageur " (voir notamment les trois "digressions théoriques" du chapitre 1 rédigé par Olivier de Sardan).

Le cœur de l'ouvrage réside précisément dans l'analyse des déclinaisons sur le terrain (dans des " contextes ») du modèle voyageur du CT. L'importance des réinterprétations et des détournements, ainsi que des décalages, divergences et contradictions, ressort particulièrement dans cette analyse des $\mathrm{CT}$ dans leurs contextes locaux. La " revanche du contexte " (expression reprise dans le titre de l'excellente introduction et du premier chapitre) exprime ainsi les multiples détournements liés à la mise en œuvre d'un modèle 
(celui du cash transfer) dans des contextes locaux. Finalement, par la démarche anthropologique, la question de la mise en œuvre est remise au centre de l'évaluation des dispositifs de développement.

Dans l'approche anthropologique, les contextes sont spécifiques, hétérogènes, selon des « configurations locales particulières, politiques, sociales, institutionnelles et de "groupes stratégiques" aux agendas multiples ". Mais, au-delà des spécificités des CT dans leurs contextes, des points critiques communs apparaissent: l'État, la rente, le ciblage, les conditionnalités, les services publics, la perspective maternaliste, l'équipe $\mathrm{CT}$, et enfin les adaptations mutuelles. Ce qui laisse entrevoir la possibilité de tirer des leçons transversales des études de cas menées, même si les auteurs rejettent toute normativité dans leurs propos ("We do not have a basic ideological position for or against CTs ", p. 20). À travers les études de cas, comme cela est souligné dans l'introduction de l'ouvrage, les problèmes classiques du développement ("La non prise en compte des contextes; les conséquences inattendues de la rente de l'aide au développement; la stigmatisation des communautés indigènes en Amérique latine; le statut incertain des bureaucrates sur le terrain et plus particulièrement des travailleurs sociaux; les illusions de multiples stratégies de participation ; l'impact des représentations de genre sur la mise en œuvre ; les dynamiques de résistance, d'acceptation, et de contournement... ") se trouvent à nouveau posés, en mettant en avant l'importance des contextes.

Pour les économistes, qu'ils s'intéressent au développement ou non, l'ouvrage est important. Par sa méthodologie et la mise en avant de l'importance des contextes dans la mise en œuvre des CT, les analyses menées font écho aux approches en termes d'économie politique (Egg et al., 2018), prenant en compte les contextes, les territoires, les études de cas dans l'analyse des politiques économiques. Se dessinent alors des points de convergence entre une économie politique du développement, ouverte à la pluridisciplinarité, et une approche anthropologique du développement. II ne s'agit pas d'imaginer une transdisciplinarité, mais bien une pluridisciplinarité où les disciplines dialoguent autour d'un objet commun, sur la base de leurs méthodes spécifiques : plusieurs études et ouvrages récents illustrent bien l'intérêt de ces approches pluridisciplinaires, menées en équipe (Bouard et al., 2016), comme les expertises collégiales de l'IRD (Le Meur et al., 2018)... Une telle pluridisciplinarité passe aussi par une réflexion sur le statut de la preuve en sciences sociales.

Du point de vue de l'économie politique du développement, il y a un consensus sur le fait que les analyses quantitatives ne suffisent pas à produire des preuves irréfutables auxquelles toute politique devrait s'adosser (evidence based policy). À cet égard, les analyses critiques des ERC (Bédécarrats et al., 2019), largement mobilisés pour justifier les $\mathrm{CT}$, continuent de nourrir un nécessaire scepticisme. Celui-ci est partagé par une large proportion des économistes s'intéressant au développement, y compris les plus reconnus (André Orléan, Robert Boyer, Philippe Hugon, Angus Deaton, Dani Rodrik, Joseph Stiglitz...), qui savent mettre à distance les résultats des travaux les plus quantitatifs et les plus théoriques, pour prendre en compte les spécificités liées à l'histoire et aux territoires (approches institutionnalistes, régulationnistes, d'économie politique...). Reconnaître les 
limites des approches quantitatives ne conduit pas pour autant à en rejeter a priori leurs apports, mais plutôt à en relativiser leur portée, parfois plus illustrative que démonstrative.

De façon réciproque, les analyses qualitatives, basées sur des terrains, contextualisées, ne peuvent remplir à elles seules le champ de la recherche en sciences sociales (le reproche de l'hégémonie de la discipline économique vaut pour les autres disciplines...). Ce que montre à nouveau l'ouvrage, c'est l'apport de l'approche anthropologique à la compréhension du changement social. Dans un contexte marqué par la diffusion d'approches quantitatives basées sur l'exploitation de bases de données de plus en plus massives, par le recours à des algorithmes purement informatiques (sans référence explicite à des théories ou méthodes des sciences sociales) au sein des différentes sciences sociales, mais plus largement au sein de l'ensemble de nos sociétés (le big data), la mise en avant des contextes et de leurs spécificités vient utilement contrebalancer cette dynamique qui questionne non seulement nos disciplines mais plus largement nos sociétés. Les possibilités d'une alternative externe aux solutions standards, telle qu'évoquée par Olivier de Sardan dans l'analyse des CT, par le fait de rendre visible les solutions (" éparpillées, discrètes, hétérogènes ") provenant du terrain, sont fortement limitées par l'extension de ces nouvelles approches quantitatives.

Les différences d'approche, de méthodologie, d'outils des disciplines anthropologiques et économiques ne sont heureusement pas destinées à disparaître. Même si cela était envisageable, ce ne serait pas souhaitable. La diversité des approches disciplinaires est une force qui peut se développer grâce à la constitution d'équipes pluridisciplinaires travaillant sur des objets de recherche communs (voir par exemple les travaux de l'IRAM et du GEMDEV). II nous semble ainsi que l'alternative interne (" one of the best strategy ") proposée par Olivier de Sardan au « modèle voyageur » du CT et à la dé-contextualisation constitue une ouverture dans cette direction, des équipes pluridisciplinaires étant certainement plus à même de créer des "fenêtres d'opportunités pour des subversions positives et des adaptations innovantes de la part des acteurs locaux ».

\section{Bibliographie}

Bédécarrats F., Guérin I., Morvant-Roux S., Roubaud F., 2019, « Estimating microcredit impact with low take-up, contamination and inconsistent data. A replication study of Crépon, Devoto, Duflo, and Pariente (American Economic Journal: Applied Economics, 2015) », IREE, n³ 3, pp. 1-53.

Bouard S., Sourisseau J.-M., Geronimi V., Blaise S., Ro'i L., Vernières M., 2016, La Nouvelle-Calédonie face à son destin : quel bilan à la veille de la consultation sur la pleine souveraineté ?, Paris, Karthala.

Egg J., Gabas J.-J., Geronimi V., Vernières M., 2018, «Essai sur l'économie politique du développement : en hommage à Philippe Hugon ", Mondes en développement, $n^{\circ} 184$, pp. 137 164.

Le Meur P.-Y., Arndt N., Christmann P., Geronimi V., 2018, " Deep-sea mining prospects in French Polynesia: Governance and the politics of time », Marine Policy, n 95, pp. 380-387. 
Olivier de Sardan J.-P., 1995, Anthropologie et développement: essai en socio-anthropologie du changement social, Marseille, Paris, APAD, Karthala.

Olivier de Sardan J.-P., 2004, "La rigueur du qualitatif. L'anthropologie comme science empirique », Espace Temps, $\mathrm{n}^{\circ}$ 84, pp. 38-50. 